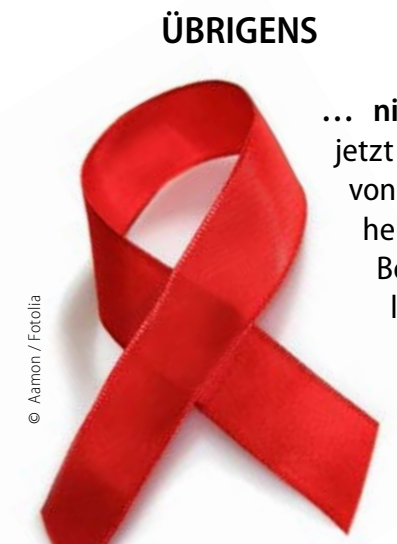

... nimmt Timothy Brown jetzt die PrEP. Brown gilt als von seiner HIV-Infektion geheilt, nachdem er zur Behandlung einer zusätzlich aufgetretenen Leukämie eine Knochenmarkspende mit mutierten Stammzellen erhalten hatte.

... ist ein aktueller Versuch, einen HIVPatienten per Stammzelltransplantation zu heilen, gescheitert. Nach genau 288 Tagen, in denen sie unter der Nachweisgrenze geblieben war, stieg die Viruslast wieder stark an.

... hat man bei HIV-Patienten mit langjährig kontrollierter Viruslast, die aus anderen Gründen gestorben sind, in $70 \%$ der Fälle HIV-DNA im Gehirn gefunden.

Komorbidität

\title{
HCV-Therapie durch DAA vereinfacht
}

Zahlreiche Präparate vom DAA-Typ (Direct-acting antiviral drugs) eröffnen bei HIV-Infizierten, die auch HCV-positiv sind, inzwischen die Möglichkeit der Interferon-freien Behandlung.

HIV-Therapeuten schätzen, dass sich in Deutschland etwa 10.000 HIV-Infizierte auch mit dem Hepatitis-C-Virus (HCV) angesteckt haben. Gemäß den aktuellen Leitlinien der Europäischen klinischen AIDS-Gesellschaft (EACS) von Anfang 2017 gilt die Kombination von Sofosbuvir mit einer an das Körpergewicht angepassten Ribavirin-Dosis über 12 Wochen als Standardtherapieregime bei Patienten mit Nachweis des HCV-Genotyps 2. Patienten mit einer Zirrhose könnten noch weitere 16 Wochen behandelt werden. Jedoch liege die Ansprechrate aktuellen Studien zufolge unter $90 \%$, weshalb akti-

\section{RKI warnt}

\section{Syphilis ist auf dem Vormarsch}

Auch 2017 haben wir in der Sommerakademie ausgewählte CME-Kurse für Ihre hausärztliche Fortbildung zusammengestellt.

Zum Thema "HIV“ finden Sie unter

http://bit.ly/2hFwgdK

aktuell den Kurs

\section{"HIV-Infektion - Test und Behandlung"}

Zur Teilnahme benötigen sie ein e.Med-Abo, mit dem sie Zugang zu allen Inhalten auf SpringerMedizin.de erhalten.

Nutzen Sie die Sommerzeit, um CME-Punkte zu sammeln und um sich schnell und effizient fortzubilden. Testen Sie SpringerMedizin.de 30 Tage lang kostenlos und unverbindlich mit dem e.Med-Abo: http://bit.ly/2vhm8el

Durch die guten Therapiemöglichkeiten hat die HIV-Infektion deutlich an Schrecken verloren. Die Kehrseite: Sexuell übertragbare Erkrankungen (STD) nehmen wieder zu. Besonders die Zahl der Syphilisfälle ist seit 2010 kontinuierlich gestiegen: Allein 2015 wurden dem RKI 6.834 neue Fälle gemeldet, 19\% mehr als im Vorjahr. Dabei wurde der größte Zuwachs bei den MSM (Männer, die Sex mit Männern haben) verzeichnet.

Nach Dr. Nils Postel, Infektiologe aus München, könnte hierbei die Einführung der PrEP eine Rolle spielen: PrEP-Nutzer neigen dazu, das Kondom, das ja nicht nur vere Kombinationen nötig seien. Als Beispiel nennt die Leitlinie Sofosbuvir plus Velpatasvir.

\section{Was empfiehlt die Leitlinie?} Antiviral drugs) vor allem gegen die HCV-Genotypen 1 und 4 eröffnet sich die Möglichkeit unter anderem einer Interferon- und Ribavirin-freien Therapie. Die Leitlinie empfiehlt vor allem die Behandlung mit Sofosbuvir plus Simeprevir bei den Genotypen (GT) 1 und 4, die Fixkombis Sofosbuvir/Ledipasvir (GT 1 und 4), Elbasvir/Grazoprevir (GT 1 und 4), Sofosbuvir/Velpatasvir (GT 1-6) sowie Sofosbuvir plus Daclatasvir (GT 1, 2, 3 und 4). Eine weitere Option sei die Kombination aus Ombitasvir/Paritaprevir/r und Dasabuvir (bei GT 1 sowie bei GT 4 ohne Dasabuvir). „r“ steht für den Booster Ritonavir.
Aufgrund neuer DAAs (Direct-Acting

vor HIV-Infektion schützt, wegzulassen. In der IPERGAY-Studie zogen sich 34\% der Teilnehmer eine STD zu [1].

Besorgniserregend sind auch die zunehmenden Resistenzen gegen Antibiotika, die bei Syphilis und Gonorrhö eingesetzt werden. Das RKI fordert, den „konsistenten Gebrauch von Kondomen bei Analverkehr unabhängig vom HIVStatus" noch stärker zu propagieren. $\mathrm{Zu}$ sätzlich sollten vor allem sexuell aktive MSM regelmäßig auf Syphilis gescreent werden, nicht zuletzt, um das Transmissionsgeschehen frühzeitig zu unterbrechen.

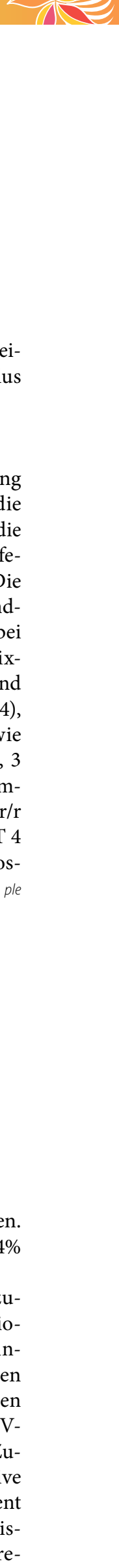

